\title{
Anatomical Characteristics and Surgical Treatments of Pincer Nail Deformity
}

\author{
Dong Ju Jung, Jae Hee Kim, Hee Young Lee, Dong Chul Kim, Se Il Lee, Tae Yeon Kim \\ Department of Plastic and Reconstructive Surgery, Bundang Jesaeng General Hospital, Seongnam, Korea
}

Background Pincer nail deformity is a transverse overcurvature of the nail. This study aimed to define the anatomical characteristics of pincer nail deformity and to evaluate the surgical outcomes.

Methods A retrospective review was conducted on 20 cases of pincer nail deformity of the great toe. Thirty subjects without pincer nail deformity or history of trauma of the feet were selected as the control group. Width and height indices were calculated, and interphalangeal angles and base widths of the distal phalanx were measured with radiography. We chose the surgical treatment methods considering perfusion-related factors such as age, diabetes mellitus, kidney disease, and peripheral vascular disease. The zigzag nail bed flap method $(n=9)$ and the inverted $T$ incision method $(n=11)$ were used to repair deformities. The outcomes were evaluated 6 months after surgery.

Results The interphalangeal angle was significantly greater in the preoperative patient group $\left(14.0^{\circ} \pm 3.6^{\circ}\right)$ than in the control group $\left(7.9^{\circ} \pm 3.0^{\circ}\right)(P<0.05)$. The postoperative width and height indices were very close to the measurements in the control group, and most patients were satisfied with the outcomes.

Conclusions We believe that the width and height indices are useful for evaluating the deformity and outcomes of surgical treatments. We used two different surgical methods for the two patient groups with respect to the perfusion-related factors and found that the outcomes were all satisfactory. Consequently, we recommend taking into consideration the circulatory condition of the foot when deciding upon the surgical method for pincer nail deformity.

Keywords Nails, ingrown / Onychomycosis / Osteophyte
Correspondence: Tae Yeon Kim Department of Plastic and Reconstructive Surgery, Bundang Jesaeng General Hospital, 20 Seohyeon-ro 180beon-gil, Bundang-gu, Seongnam 463-774, Korea

Tel: +82-31-779-0281

Fax: +82-31-779-0164

E-mail: kty@dmc.or.kr

No potential conflict of interest relevant to this article was reported.

Received: 13 Sep 2014 • Revised: 4 Dec 2014 • Accepted: 23 Dec 2014

pISSN: 2234-6163 • elSSN: 2234-6171 • http://dx.doi.org/10.5999/aps.2015.42.2.207• Arch Plast Surg 2015;42:207-213

\section{INTRODUCTION}

Pincer nail deformity was first described by Cornelius and Shelley [1] in 1968 as a transverse distal overcurvature of the nail (Fig. 1). This deformity may cause cosmetic problems as well as pain and discomfort when walking or wearing shoes. The great toe nails are usually affected, but other toes and fingers may also be involved [2]. In the past, pincer nail deformity was considered a type of ingrown nail rather than being clearly classified as a separate disease. In ingrown nail, the nail plate pierces the lateral nail fold, which may cause foreign body reactions and infection. However, in contrast to the shape of the nail plate in pincer nail deformity, that in an ingrown nail is normal $[3,4]$.

Pincer nail deformity can have a hereditary or acquired origin, 


\section{Fig. 1. Various pincer nails removed from toes}

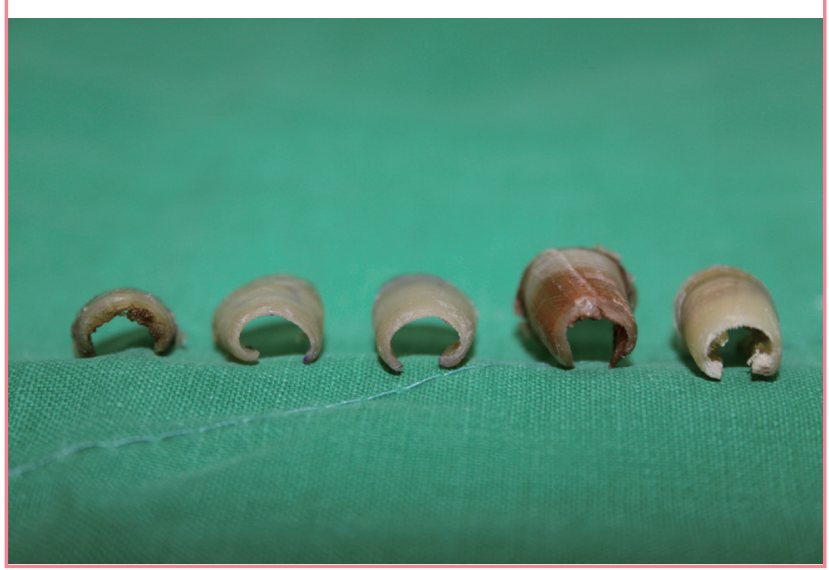

and associations with ill-fitting shoes, osteoarthritis, onychomycosis, or use of $B$-blockers have been reported [5-7]. A number of treatment approaches have been developed for this condition. Although conservative treatment, which may include nail grinding, a nail brace, and application of urea paste, is simple and convenient for the patient, the high rate of recurrence often requires long-term treatment, and the effect may be modest in cases with severe deformity [5,8-10]. Further, most patients seek medical help because of discomfort or pain caused by pincer nail deformity rather than because of its cosmetic appearance. Therefore, in a typical case, the deformity has already severely progressed by the time surgical treatment is indicated.

The aim of this study is to analyze the anatomical characteristics of the nail and phalangeal bone of pincer nail deformity by conducting nail measurements and a radiologic study including comparisons with a normal control group. In addition, although many types of treatment are used for pincer nail deformity, few published papers have reported on the results of these treatments.

In this study, we applied two different surgical methods depending on the deformity's indications and compared and analyzed their results.

\section{METHODS}

\section{Patients}

We conducted a retrospective review of 20 cases (14 patients) of pincer nail deformity of the great toe that were surgically treated between August 2011 and October 2013 at our department. Patient age, sex, duration of disease, medical history, familial history of pincer nail deformity, presence of onychomycosis infection, and surgical methods were reviewed, as well as preoperative and postoperative measurements of the nail plate and the results of a radiologic examination. Thirty subjects without pincer nail deformity or history of trauma of the feet were selected

\section{Fig. 2. Illustration of the nail measurement}

$A$, width of the nail root; $B$, width of the nail tip; $C_{1}$ height of the nail tip. Width index $=B / A \times 100$, height index $=C / B \times 100$

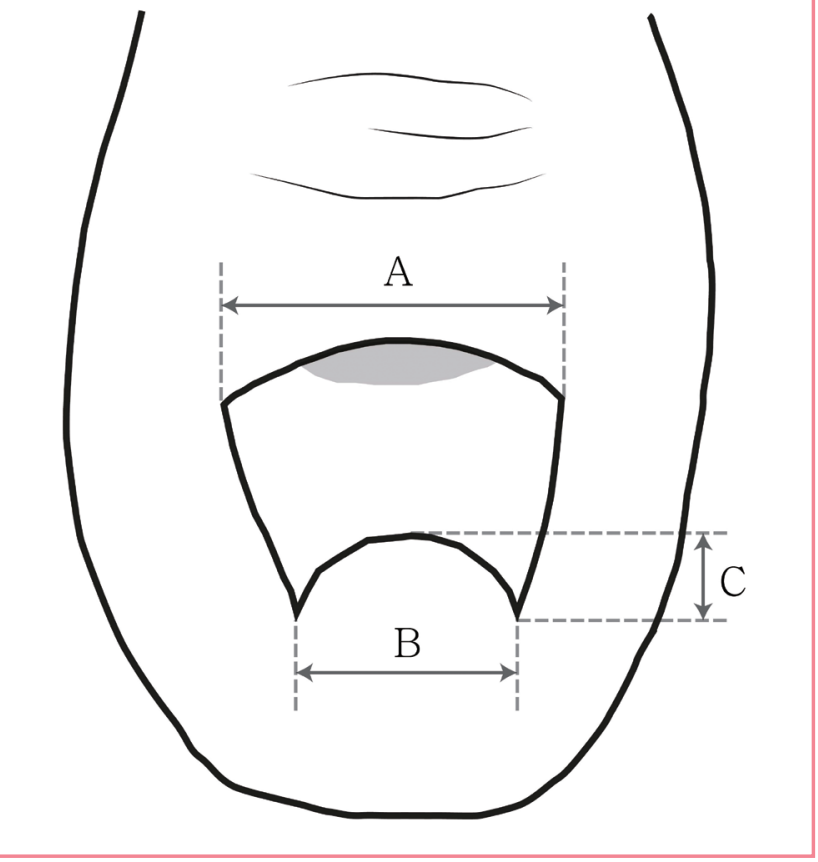

as the control group ( 15 men and 15 women, both great toes: $\mathrm{n}=60)$.

\section{Assessment methods}

\section{Measurement of the nail plate}

The nail plate was evaluated by measuring the widths of the nail root and nail tip and the height of the nail tip, followed by calculation of the width and height indices. The width index is defined as the percentage ratio of the width of the nail tip to the width of the nail root, whereas the height index is defined as the percentage ratio of the height of the nail tip to the width of the nail tip (Fig. 2) [11]. The abovementioned measurements were conducted in the control and patient groups before and 6 months after the surgical treatment.

\section{Radiologic evaluation}

Lateral view radiography of the great toe was performed with hyperextension to identify osteophytes. We measured the interphalangeal angle for the evaluation of the lateral deviation of the great toe. The interphalangeal angle between the long axes of the distal and proximal phalanxes was measured on anteroposterior radiographs (Fig. 3). Baran et al. [5] suggested that pincer nail deformity is a result of an enlargement of the base width of the distal phalanx, which increases the curvature of the distal part of the nail plate. Therefore, we compared the base widths of 


\section{Fig. 3. Illustration of the measurement on X-ray}

Interphalangeal angle and base width of the distal phalanx in the (A) control and (B) patient groups.
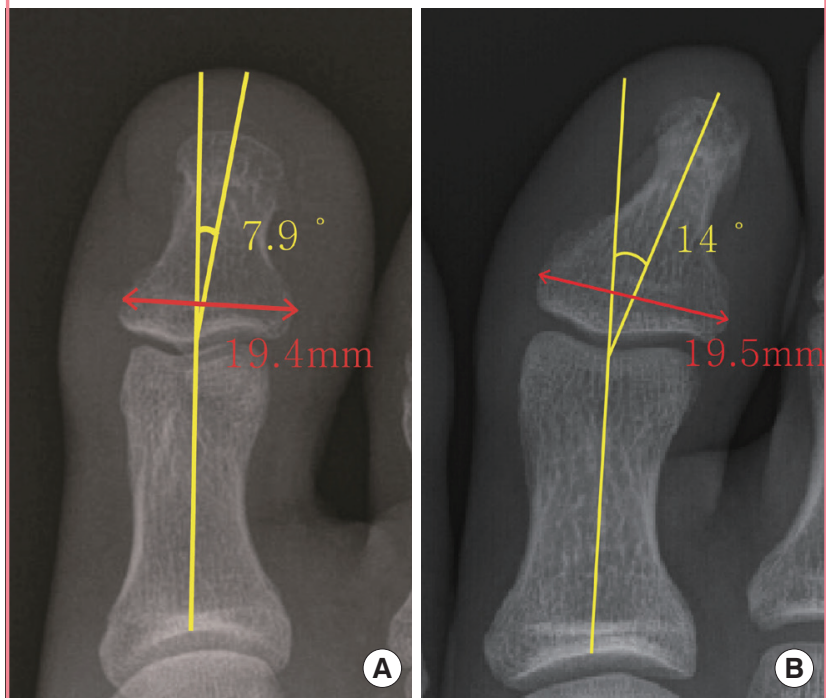

the distal phalanxes in the patient and control groups by using anteroposterior radiographs. The base widths of the distal phalanxes is defined as the maximum length of the base of the distal phalanx, which is parallel to the imaginary line between the lateral and the medial end points of the articular surface of distal phalanxes.

\section{Surgical technique}

A digital block was performed by injecting $1 \%$ lidocaine, and a rubber tourniquet was applied. The deformed nail plate was removed from the great toe by using an elevator without inducing nail bed injury. In this study, two surgical methods were employed. We performed the inverted $\mathrm{T}$ incision method, which is less invasive than the zigzag nail bed flap method, in the vascular high-risk group including patients such as elderly patients more than 70 years of age and patients with diabetes mellitus, chronic kidney disease, and/or peripheral vascular disease. We performed the zigzag nail bed flap method in 9 patients, and the inverted $\mathrm{T}$ incision method in 11 patients.

\section{Zigzag nail bed flap method [12]}

A W-shaped incision was made with a nail margin of $5 \mathrm{~mm}$. The nail bed flap was elevated from the distal phalangeal bone by subperiosteal dissection with an elevator. The exposed dorsally protruding osteophyte was removed with a diamond burr. Partial excision of the hyponychium of the nail bed flap was performed, and a "double w"-shaped incision was made. The lateral nail walls were de-epithelialized, and the widened nail bed flap was sutured with 4-0 nylon (Fig. 4).

\section{Fig. 4. Zigzag nail bed flap method}

(A) Preoperative photograph. A W-shaped incision is made with a nail margin of $5 \mathrm{~mm}$. (B) The nail bed flap is elevated, and the exposed dorsally protruding osteophyte is removed using a diamond burr. (C) The hyponychium is partially excised, and the lateral nail walls are de-epithelialized. (D) The widened nail bed flap is sutured using 4-0 nylon. (E) An image taken at the 6-month follow-up visit.
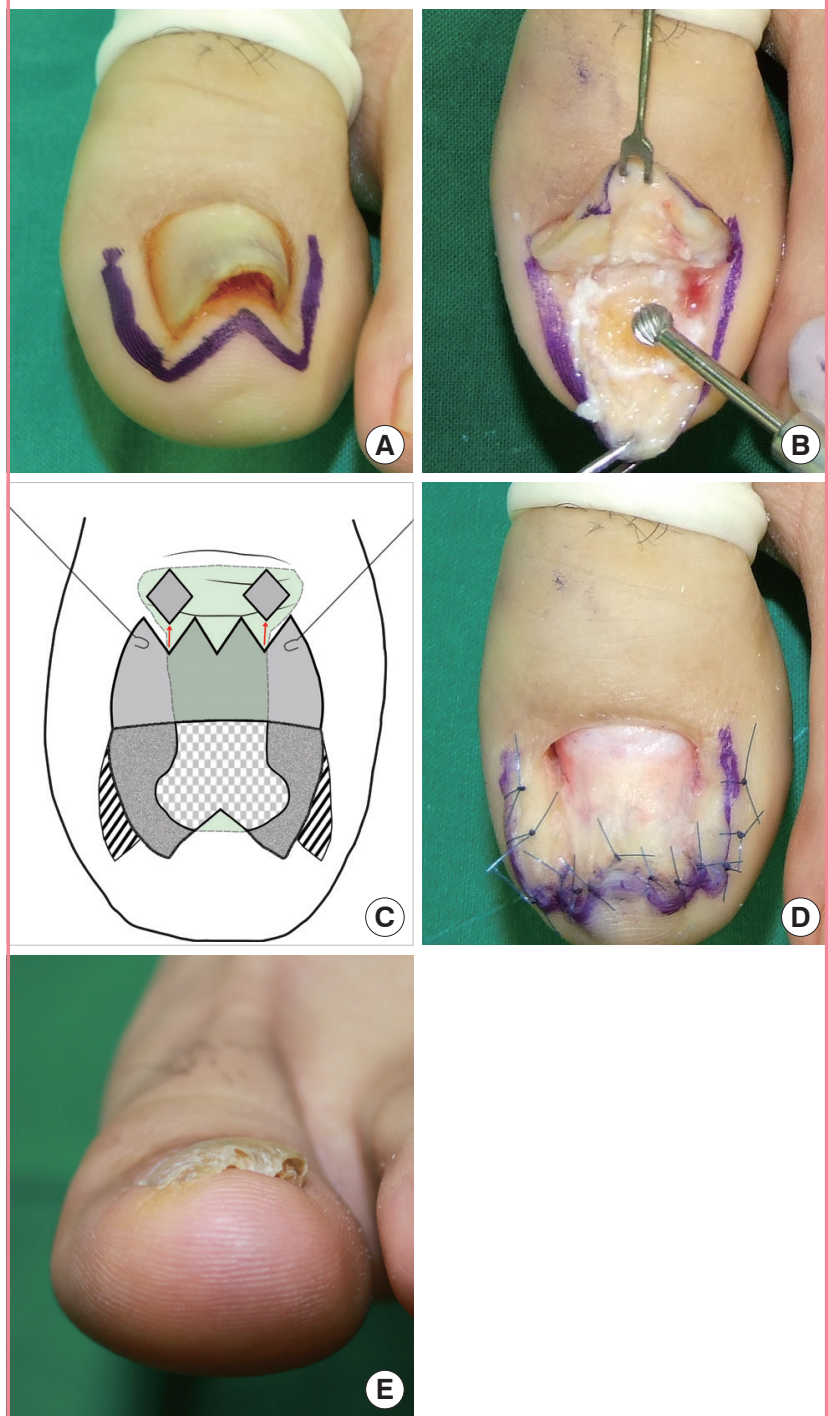

Inverted T incision method (modified Haneke's method) [5] We modified Haneke's method by using an inverted $\mathrm{T}$ incision instead of a median longitudinal incision. To expose the osteophyte appropriately, the inverted $\mathrm{T}$ incision was made down to the bone. After the nail bed flap was elevated, the exposed protruding osteophyte was removed using a diamond burr, and the nail bed was repaired. The bilateral matrix horn was electrocauterized. Lastly, all the incision sites were sutured with 4-0 nylon. In contrast to Haneke's method, a tie-under suture was not applied and the osteophyte was removed with a diamond burr instead of a rongeur (Fig. 5). 


\section{Fig. 5. Inverted T incision method}

(A) Preoperative photograph. (B) An inverted T incision is made down to the bone, and an oblique incision is made at the junction of the proximal and lateral nail folds. (C) The nail bed flap is elevated, and the dorsally protruding osteophyte is removed. The bilateral matrix horn is electrocauterized. All incision sites are repaired using 4-0 nylon. (D) An image taken at the 6-month follow-up visit.
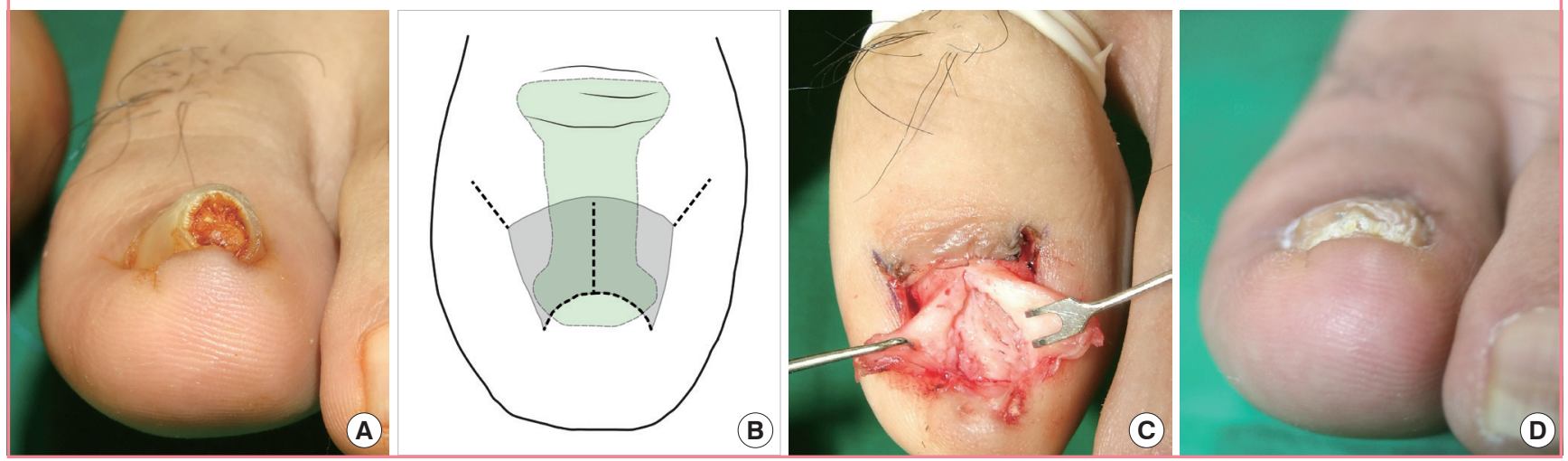

After completing the abovementioned two surgical procedures, we applied a silastic sheet on the nail bed and fixed it using 4-0 nylon. Antibiotics and non-steroidal anti-inflammatory drugs were prescribed for 5 days, and the stitches were removed after 2 weeks.

\section{Patient satisfaction}

Six months after the operation, assessment of patient satisfaction with the treatment was conducted using a questionnaire with scores ranging from 1 to 5 (1, very dissatisfied; 2, dissatisfied; 3 , fair; 4 , satisfied; 5 , very satisfied). The questionnaire evaluated the newly grown nail appearance (contour, thickness, irregularity, fragility, and color) as well as pain and discomfort when walking.

\section{RESULTS}

\section{Demographics}

The patient group consisted of 6 men and 8 women with the median age of 58 years (range, 19-90 years) (Table 1). It was bilateral in 7 patients and unilateral in the other 7 patients. Five patients had a family history of pincer nail deformity. All patients had a family history of a bilateral deformity. The disease duration ranged from 1 to 20 years, with a median of 3.5 years. Seven patients had underlying disease, including chronic kidney disease, hypertension, diabetes mellitus, Buerger's disease, and angina. One of the seven patients who had bilateral deformity underwent surgical treatment for deformity on only one side, and the other 6 patients underwent surgical treatment for both sides. Onychomycosis infection was present in 4 out of 20 nail plates. The postoperative follow-up period ranged from 6 to 24 months, with a median of 8.5 months.
Table 1. Demographics and clinical data of 14 patients with pincer nail deformities

\begin{tabular}{|lc|}
\hline Characteristic & \\
\hline Gender (male:female) & $6: 8$ \\
No. of toes (unilateral:bilateral) & 20 (7:14) \\
Median age (yr) & 58 (range, 19-90) \\
Median disease duration (yr) & 3.5 (range, 1-20) \\
Presence of familial history & 5 \\
Unilateral deformity & 0 \\
Bilateral deformity & 5 \\
Absence of familial historya) & 9 \\
Unilateral deformity & 7 \\
Bilateral deformity & 2 \\
Onychomycosis infection (\%) & $20(4 / 20)$ \\
Osteophyte on radiographs (\%) & $70(14 / 20)$ \\
Median follow-up period (mo) & $8.5($ (range, 6-24) \\
\hline alNo. of patients. & \\
\hline
\end{tabular}

\section{Nail measurements}

The mean proximal nail width, distal nail width, and distal nail height of the male subjects in the control group $(n=15)$ were $16.5 \pm 1.5 \mathrm{~mm}, 16.1 \pm 1.5 \mathrm{~mm}$, and $3.3 \pm 0.7 \mathrm{~mm}$, respectively. The corresponding values were $13.9 \pm 1.1 \mathrm{~mm}, 13.8 \pm 1.2 \mathrm{~mm}$, and $3.3 \pm 1.0 \mathrm{~mm}$ for the female controls $(\mathrm{n}=15)$. Although the proximal distal widths were significantly higher in the men than in the women $(\mathrm{P}<0.05)$, there were no statistically significant differences in the width and height indices $(\mathrm{P}>0.05)$ (Table 2). The mean width index in the patient group was 1.6 -fold lower than that in the control group, whereas the mean height index in the patient group was about 3.5 times greater.

\section{Radiologic study}

Lateral radiographs revealed osteophytes in 13 out of 60 cases (21.6\%) in the control group (4 in men [13.3\%] and 9 in the 
women [30\%]). In contrast, osteophytes were detected in 14 out of 20 cases $(70 \%)$ in the pincer nail deformity patients.

In the control group, the mean interphalangeal angles between the distal and proximal phalanxes of the great toe were $8.6^{\circ} \pm 3.3^{\circ}$ in the men and $7.2^{\circ} \pm 2.6^{\circ}$ in the women, with no statistically significant difference between these values. The mean interphalangeal angle in the patient group was significantly higher than that in the control group $\left(14.0^{\circ} \pm 3.6^{\circ}\right.$ vs. $\left.7.9^{\circ} \pm 3.0^{\circ}, \mathrm{P}<0.0 .5\right)$. The base width of the distal phalanx showed no significant difference between the control and the patient groups (19.4 \pm 1.5 $\mathrm{mm}$ and $19.5 \pm 1.6 \mathrm{~mm}$, respectively) (Table 3 ).

\section{Treatment results}

The nail plates were assessed 6 months after surgery, with the exception of one case where the toenail did not grow completely. The mean width and height indices of $89.4 \pm 3.9$ and $22.7 \pm 5.5$, respectively, were very close to the measurements in the control group. Thus, the width index increased by $49.5 \%$ and the height index decreased by $71.6 \%$, indicating a substantial improvement in the nail shape as a result of the treatment. There were no statistically significant differences in the indices between the patients treated with the zigzag nail bed flap method and the inverted $\mathrm{T}$ incision method (Table 4).

Assessment of patient satisfaction revealed the scores of 4 for the newly grown nail and 4.2 for the ease of walking. The corresponding scores were 3.9 and 4.1 in the group treated with the zigzag flap nail bed flap method and 4.1 and 4.3 in the group treated with the inverted $\mathrm{T}$ incision method.

\section{Complications and recurrence}

The following patient was our first case of this study and was presented before the abovementioned surgical indications regarding perfusion-related factors were established. The nail plate grew incompletely in a 71-year-old woman treated with the zigzag nail bed flap method. This patient was diagnosed with diabetes mellitus 25 years before the treatment and was undergoing dialysis for chronic kidney disease. Ischemic changes were

Table 3. Radiologic study in the control group and the pincer nail deformity group

\begin{tabular}{lccc}
\hline Radiologic finding & $\begin{array}{c}\text { Control group } \\
(\mathbf{n}=\mathbf{6 0})\end{array}$ & $\begin{array}{c}\text { PND group } \\
(\mathbf{n}=\mathbf{2 0})\end{array}$ & P-value $^{\text {a) }}$ \\
\hline Osteophyte $(+)^{b)}$ & $13(21.6)$ & $14(70)$ & - \\
Interphalangeal angle $\left(^{\circ}\right)$ & $7.9 \pm 3.0$ & $14.0 \pm 3.6$ & $<0.05$ \\
Base width $(\mathrm{mm})$ & $19.4 \pm 1.5$ & $19.5 \pm 1.6$ & 0.694
\end{tabular}

Values are presented as number (\%) or mean \pm standard deviation. PND, pincer nail deformity.

a)T-test; b) Osteophyte was detected on X-ray.

Table 2. Nail plate measurements in the control group and the pincer nail deformity group before the treatment

\begin{tabular}{lccccc}
\hline Group & $\begin{array}{c}\text { Width of the nail } \\
\text { root }(\mathbf{m m})\end{array}$ & $\begin{array}{c}\text { Width of the nail } \\
\text { tip }(\mathbf{m m})\end{array}$ & $\begin{array}{c}\text { Height of the nail } \\
\text { tip }(\mathbf{m m})\end{array}$ & $\begin{array}{c}\text { Width index } \\
(\%)\end{array}$ & $\begin{array}{c}\text { Height index } \\
(\%)\end{array}$ \\
\hline Control group $(n=60)$ & $15.2 \pm 1.9$ & $14.9 \pm 1.8$ & $3.3 \pm 0.9$ & $98.3 \pm 5.3$ & $22.4 \pm 7.4$ \\
Men $(n=30)$ & $16.5 \pm 1.5$ & $16.1 \pm 1.5$ & $3.3 \pm 0.7$ & $97.2 \pm 3.4$ & $20.5 \pm 5.2$ \\
Women $(n=30)$ & $13.9 \pm 1.1$ & $13.8 \pm 1.2$ & $3.3 \pm 1.0$ & $99.3 \pm 6.6$ & $24.4 \pm 8.8$ \\
PND group $(n=20)$ & $14.8 \pm 1.9$ & $8.8 \pm 1.9$ & $6.7 \pm 1.9$ & $59.8 \pm 12.5$ & $80.0 \pm 29.5$ \\
\hline
\end{tabular}

Values are presented as mean \pm standard deviation.

PND, pincer nail deformity.

\section{Table 4. Nail plate measurements in the pincer nail deformity group before and 6 months after the treatment}

\begin{tabular}{|c|c|c|c|c|c|}
\hline Group & $\begin{array}{l}\text { Width of the nail } \\
\text { root }(\mathrm{mm})\end{array}$ & $\begin{array}{l}\text { Width of the nail } \\
\text { tip }(\mathrm{mm})\end{array}$ & $\begin{array}{l}\text { Height of the nail } \\
\text { tip }(\mathrm{mm})\end{array}$ & $\begin{array}{l}\text { Width index } \\
(\%)\end{array}$ & $\begin{array}{l}\text { Height index } \\
(\%)\end{array}$ \\
\hline \multicolumn{6}{|c|}{ PND group $(n=20)$} \\
\hline Preoperative & $14.8 \pm 1.9$ & $8.8 \pm 1.9$ & $6.7 \pm 1.9$ & $59.8 \pm 12.5$ & $80.0 \pm 29.5$ \\
\hline Postoperative & $14.8 \pm 1.8$ & $13.2 \pm 1.3$ & $3.0 \pm 0.7$ & $89.4 \pm 3.9$ & $22.7 \pm 5.5$ \\
\hline \multicolumn{6}{|c|}{ Zigzag nail bed flap method $(n=9)$} \\
\hline Preoperative & $14.1 \pm 1.9$ & $8.5 \pm 1.8$ & $6.9 \pm 1.4$ & $60.4 \pm 10.1$ & $85.4 \pm 30.4$ \\
\hline Postoperative & $14 \pm 1.4$ & $12.7 \pm 1.2$ & $3.1 \pm 0.8$ & $90.6 \pm 3.1$ & $25.0 \pm 6.3$ \\
\hline \multicolumn{6}{|c|}{ Inverted T incision method $(n=11)$} \\
\hline Preoperative & $15.3 \pm 1.9$ & $9.0 \pm 2.1$ & $6.5 \pm 2.2$ & $59.3 \pm 14.7$ & $75.6 \pm 29.3$ \\
\hline Postoperative & $15.4 \pm 1.9$ & $13.6 \pm 1.3$ & $2.8 \pm 0.6$ & $88.5 \pm 4.2$ & $21.0 \pm 4.4$ \\
\hline
\end{tabular}

Values are presented as mean \pm standard deviation.

One patient's nail did not grow back completely, so she was excluded from the measurement at 6 months after the treatment. PND, pincer nail deformity. 
detected on the nail bed flap of this patient after the operation. However, after 4 months of conservative treatment, the nail plate started to grow, and the color of the nail bed flap improved. In five out of the nine great toes for which the zigzag nail bed flap method was used, sensory disturbance developed around the incision site, but this symptom gradually improved.

No other complications, such as infection, hematoma, or dehiscence, were observed, and pincer nail deformity did not recur.

\section{DISCUSSION}

Most of the patients considered in this study visited our clinic because of various degrees of discomfort and pain during daily activities or when walking rather than the cosmetic appearance of their toenails. This explains a higher than expected mean duration of pincer nail deformity of 5.2 years and the advanced stage of the disease, with the width index 1.6 times lower and the height index 3.5 times higher than in the control group. Out of the 14 pincer nail deformity patients, 5 (35.7\%) had a family history of this disease. Of note, all these patients had bilateral deformity, which is in agreement with the conclusions of other studies that hereditary pincer nail deformity usually occurs bilaterally [13].

In pincer nail deformity, the toe nail curvature increases from the nail root to the nail tip, and the nail height is increased at the nail tip area. Although DuVries [14] suggested that deformity is preceded by the formation of an osteophyte, it is now widely accepted that nail deformity develops first. In the study conducted by Kosaka et al. [4], osteophytes were present in 10 out of 20 normal people, and there was no correlation between the nail height and the osteophyte height, which implies that osteophytes developed as a result of the pincer nail deformity. According to Plusjé [2], this appositional bone growth is due to the distal part of the nail plate curving upward. Furthermore, an investigation of cadaver specimens showed that the shape of the distal nail plate is not affected by the phalangeal shape but might be influenced by some intrinsic and extrinsic factors [15].

Radiography was routinely employed by many investigators to identify osteophytes preoperatively. In particular, osteophytes were identified in all 20 pincer nail deformity patients by Kosaka et al. [4]. According to another report, osteophytes were not identified in every pincer nail deformity patient using radiography, but they were found in $66.7 \%$ of the cases during surgery [16]. In our study, although radiography detected osteophytes in 14 out of 20 cases (70\%) of pincer nail deformity, surgery revealed that osteophytes were present in all 20 . Therefore, although radiography is useful for preoperative detection of osteophytes, its sensitivity is rather low.

The pathophysiology of pincer nail deformity has not been firmly established. Baran et al. [5] suggested that the base of the distal phalanx, which is strongly bound to the matrix by collagen fibers, becomes enlarged, and this may cause the overcurvature. Kosaka et al. [4] proposed that an unknown factor triggers nail bed shrinkage, constriction, or both, leading to the shrinkage of the ventral but not the dorsal side of the nail plate. This difference results in the formation of inward curvature. It was also proposed that deformation of the nail plate causes ischemia and inflammation of the nail bed, which in severe cases may be accompanied by scar formation and contracture, further exacerbating the deformity [12]. In our study, the base width of the distal phalanx in the pincer nail deformity group was comparable to that in the control group, but the interphalangeal angles were significantly greater. This difference may be related to the development of pincer nail deformity. Therefore, to prevent the development or recurrence of pincer nail deformity, patients and individuals with a family history of this disease should avoid wearing ill-fitting shoes or high heels, which may cause deviation of the great toe [12].

Despite the many conservative and surgical treatments available for pincer nail deformity, no standard treatment approach has been established. Surgical methods include skin grafting or mucosal grafting of the nail bed, implantation of subcutaneous tissue beneath the nail bed, Haneke's technique, and the zigzag nail bed flap method [5,12,17-19]. The latter two approaches were used in the present study. Among them, the zigzag nail bed flap method directly expands the distal nail bed flap, which makes it especially effective in cases of hardened, contracted hyponychium tissue. However, sensory disturbance of the wound margin could develop, and wound healing or nail growth could be delayed [12]. In our study, both wound healing and nail growth were remarkably slow in a 71-year-old female patient with diabetes mellitus and chronic kidney disease. This suggests that the zigzag nail bed flap method may be associated with complications in older patients and smokers, as well as in patients with diabetes mellitus, chronic kidney disease, and/or peripheral vascular disease that compromises peripheral circulation. Based on this experience, we established surgical indications regarding perfusion-related factors and applied them from the second case onwards in our study.

We introduced some modifications to Haneke's surgical method [5]. In particular, inverted $\mathrm{T}$ incisions instead of median longitudinal incisions were made in the nail bed to better expose and completely remove the osteophyte. Further, the original Haneke's method includes the application of the tie-under suture to the nail bed for expansion, which is to be kept for 3 weeks. We did not use the tie-under suture in our patients because this could cause persistent pain and dehiscence owing to excessive tension in the suture area. Finally, we opted to use a diamond 
burr instead of a rongeur for the removal of the osteophyte, resulting in a more even bone surface.

To widen the nail bed, Kosake et al. made zigzag nail bed flaps, whereas Haneke opted to utilize the tie-under suture. Although we did not use the tie-under suture in the inverted $\mathrm{T}$ incision method, satisfactory results were achieved with both approaches. Thus, the preoperative and postoperative width and height indices of the nail plate did not differ significantly between the two surgical groups. However, in cases with severe nail bed tissue deformity and scar contracture, we recommend nail bed widening procedures for better surgical outcomes.

Although many treatment methods for pincer nail deformity have been reported, few of them are based on objective measurements such as width and height indices. In the study conducted by Lee et al. [20], the mean width and height indices of 11 patients who underwent entire nail surface grinding except for the nail margin improved from $72.7 \pm 13.0$ to $87.4 \pm 10.5$ and from $58.8 \pm 29.1$ to $26.0 \pm 12.7$, respectively. In another study that used a modified Haneke's method to treat 6 patients, the preoperative mean width and height indices of 15.4 and 63.9 improved to 87.1 and 11.9, respectively [16]. In our study, the preoperative mean width index of $59.8 \pm 12.5$ increased to 89.4 \pm 3.9 as a result of the operation, whereas the mean height index decreased from $80.0 \pm 29.5$ to $22.7 \pm 5.5$. Hence, we successfully repaired severe nail deformities in our patients.

In conclusion, we compared the width and height indices between the patient and control groups and used these indices for the evaluation of surgical outcomes. We believe that the width and height indices are useful for evaluating the deformity and outcomes of surgical treatments. In the treatment results, we achieved satisfactory outcomes with both the surgical methods that included osteophyte removal. We believe that it is essential to remove osteophytes, particularly in patients with pincer nail deformity that is refractory to conservative approaches, as well as in those with advanced stage disease. In addition, we used two different surgical methods for the two patient groups with respect to perfusion-related factors and found that the outcomes were all satisfactory. Consequently, we recommend the consideration of the circulatory condition of the foot when deciding on the surgical method for pincer nail deformity.

\section{REFERENCES}

1. Cornelius CE 3rd, Shelley WB. Pincer nail syndrome. Arch Surg 1968;96:321-2.

2. Plusje LG. Pincer nails: a new surgical treatment. Dermatol Surg 2001;27:41-3.
3. Persichetti P, Simone P, Li Vecchi G, et al. Wedge excision of the nail fold in the treatment of ingrown toenail. Ann Plast Surg 2004;52:617-20.

4. Kosaka M, Kusuhara H, Mochizuki Y, et al. Morphologic study of normal, ingrown, and pincer nails. Dermatol Surg 2010;36:31-8.

5. Baran R, Haneke E, Richert B. Pincer nails: definition and surgical treatment. Dermatol Surg 2001;27:261-6.

6. Greiner D, Schofer H, Milbradt R. Reversible transverse overcurvature of the nails (pincer nails) after treatment with a beta-blocker. J Am Acad Dermatol 1998;39:486-7.

7. Higashi N. Pincer nail due to tinea unguium. Hifu 1990;32: 40-4.

8. el-Gammal S, Altmeyer P. Successful conservative therapy of pincer nail syndrome. Hautarzt 1993;44:535-7.

9. Fraser AR. Orthonyx: theory and practice. Br J Chirop 1967; 32:229-32.

10. Maeda N, Mizuno N, Ichikawa K. Nail abrasion: a new treatment for ingrown toe-nails. J Dermatol 1990;17:746-9.

11. Kosaka M, Kamiishi H. New strategy for the treatment and assessment of pincer nail. Plast Reconstr Surg 2003;111: 2014-9.

12. Kosaka M, Asamura S, Wada Y, et al. Pincer nails treated using zigzag nail bed flap method: results of 71 toenails. Dermatol Surg 2010;36:506-11.

13. Chapman RS. Letter: Overcurvature of the nails: an inherited disorder. Br J Dermatol 1973;89:317-8.

14. DuVries HL. Diseases and deformities of the toenails. In: Inman VT, editor. Surgery of the foot. St. Louis: CV Mosby; 1959. p.204-22.

15. Parrinello JF, Japour CJ, Dykyj D. Incurvated nail. Does the phalanx determine nail plate shape? J Am Podiatr Med Assoc 1995;85:696-8.

16. Moon HS, Son SJ, Park K, et al. A clinical study of efficacy of surgical management for pincer nails. Korean J Dermatol 2009;47:509-15.

17. Suzuki K, Yagi I, Kondo M. Surgical treatment of pincer nail syndrome. Plast Reconstr Surg 1979;63:570-3.

18. Hatoko M, lioka $\mathrm{H}$, Tanaka A, et al. Hard-palate mucosal graft in the management of severe pincer-nail deformity. Plast Reconstr Surg 2003;112:835-9.

19. Brown RE, Zook EG, Williams J. Correction of pincer-nail deformity using dermal grafting. Plast Reconstr Surg 2000; 105:1658-61.

20. Lee JI, Lee YB, Oh ST, et al. A clinical study of 35 cases of pincer nails. Ann Dermatol 2011;23:417-23. 